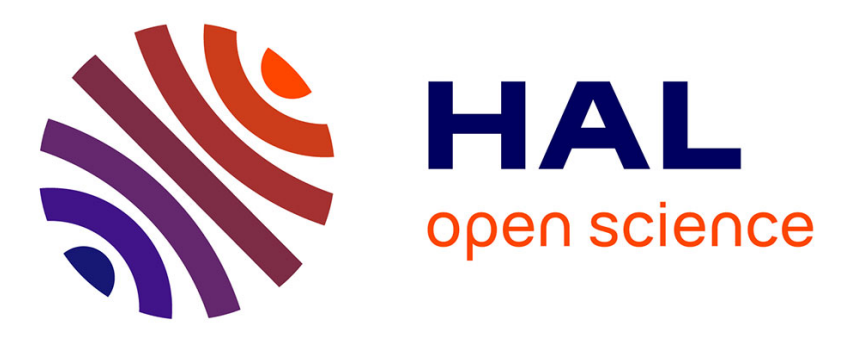

\title{
Promenade dans les mondes vécus. Les animaux peuvent-ils être des interlocuteurs de l'enquête socio-anthropologique?
}

\author{
Antoine Doré
}

\section{- To cite this version:}

Antoine Doré. Promenade dans les mondes vécus. Les animaux peuvent-ils être des interlocuteurs de l'enquête socio-anthropologique?. Droit des sociétés , 2010, 108, pp.33-45. 10.3917/soc.108.0033 . hal-02660298

\section{HAL Id: hal-02660298 \\ https: / hal.inrae.fr/hal-02660298}

Submitted on 30 May 2020

HAL is a multi-disciplinary open access archive for the deposit and dissemination of scientific research documents, whether they are published or not. The documents may come from teaching and research institutions in France or abroad, or from public or private research centers.
L'archive ouverte pluridisciplinaire HAL, est destinée au dépôt et à la diffusion de documents scientifiques de niveau recherche, publiés ou non, émanant des établissements d'enseignement et de recherche français ou étrangers, des laboratoires publics ou privés. 


\section{PROMENADE DANS LES MONDES VÉCUS. LES ANIMAUX PEUVENT- ILS ÊTRE DES INTERLOCUTEURS DE L'ENQUÊTE SOCIO- ANTHROPOLOGIQUE ?}

\section{Antoine Doré}

De Boeck Supérieur | «Sociétés »

$2010 / 2 n^{\circ} 108$ | pages 33 à 45

ISSN 0765-3697

ISBN 9782804161569

Article disponible en ligne à l'adresse :

http://www.cairn.info/revue-societes-2010-2-page-33.htm

\section{Pour citer cet article :}

Antoine Doré, « Promenade dans les mondes vécus. Les animaux peuvent-ils être des interlocuteurs de l'enquête socio-anthropologique ? », Sociétés 2010/2 (n 108), p. 33-45.

DOI $10.3917 /$ soc.108.0033

Distribution électronique Cairn.info pour De Boeck Supérieur.

(C) De Boeck Supérieur. Tous droits réservés pour tous pays.

La reproduction ou représentation de cet article, notamment par photocopie, n'est autorisée que dans les limites des conditions générales d'utilisation du site ou, le cas échéant, des conditions générales de la licence souscrite par votre établissement. Toute autre reproduction ou représentation, en tout ou partie, sous quelque forme et de quelque manière que ce soit, est interdite sauf accord préalable et écrit de l'éditeur, en dehors des cas prévus par la législation en vigueur en France. Il est précisé que son stockage dans une base de données est également interdit. 


\title{
PROMENADE DANS LES MONDES VÉCUS. LES ANIMAUX PEUVENT-ILS ÊTRE DES INTERLOCUTEURS DE L'ENQUEETE SOCIO-ANTHROPOLOGIQUE ?
}

Antoine DORÉ 1

\begin{abstract}
Résumé : Partant de l'intuition d'un rapprochement possible entre la formulation du projet de J. v. Uexküll et celles de certains sociologues et anthropologues, cet article propose d'expérimenter une lecture " par la sociologie » du travail du biologiste afin de réinterroger la place accordée aux animaux dans l'analyse des problèmes et des controverses auxquels ils participent. Après un rapide retour sur les sciences de la nature et les sciences sociales qui se sont saisies des animaux comme objet d'étude, nous mettons en évidence l'existence d'un partage épistémologique qui ne rend pleinement justice ni aux animaux des biologistes, ni aux humains des socio-anthropologues. Il s'agit alors de prendre au sérieux les relations des animaux aux mondes qui les entourent pour contribuer à la définition d'animaux réellement parties prenantes des collectifs d'humains et de non-humains qui prennent forme avec et autour d'eux.
\end{abstract}

Mots clés : interdisciplinarité, méthodologie, mondes animaux, signification, symétrie, Uexküll.

\begin{abstract}
Starting from the intuition of a possible rapprochement between the formulation of the J. v. Uexküll's project and those of some sociologists and anthropologists, this paper proposes a "sociologic reading" of the work of the biologist in order to re-examine the place given to animals in the analysis of problems and controversies in which they participate. After a quick return on the natural sciences and social sciences that have seized the animals as objects of study, we emphasize the existence of an epistemological division that does full justice neither to animals of biologists, nor to humans of socio-anthropologists. We
\end{abstract}

1. Cemagref (UR DTGR) ; Sciences Po (CSO, CNRS) ; Université de Liège (SEED).

Domaine Universitaire, 2 rue de la Papeterie - BP 76, 38402 Saint-Martin d'Hères Cedex, France.dore.antoine@yahoo.fr 
attempt to take seriously the relationship of animals with the worlds around them to contribute to a definition of animals really gone-taking of the collectives of humans and nonhumans that are taking shape with and around them.

Keywords : interdisciplinarity, méthodologie, animals worlds, signifiance, symétrique, Uexküll.

\section{Introduction}

"Ce petit livre n'a pas la prétention de servir de guide à une nouvelle science. Il s'en tient d'abord à ce que l'on peut nommer la description d'une promenade dans des mondes inconnus »2. Telles sont les premières phrases de Jacob von Uexküll dans l'avant-propos de son ouvrage Mondes animaux et monde humain. Cet article n'a pas non plus prétention à servir de guide à une nouvelle science. Partant de l'intuition d'un rapprochement possible entre la formulation du projet du biologiste et celles de certains sociologues et anthropologues ${ }^{3}$, l'objectif est d'expérimenter une lecture "par la sociologie » du travail de J. v. Uexküll afin de réinterroger la place accordée aux animaux dans l'analyse des problèmes et des controverses auxquels ils participent. La question n'est pas ici d'opérer une énième tentative de caractérisation de ce qui serait le propre de l'Homme ou de l'Animal mais plutôt d'explorer de nouvelles pistes pour l'analyse empirique des relations anthropozoologiques. Nous verrons dans un premier temps ce que l'épistémologie moderne a fait des animaux et comment ces derniers se sont trouvés écartelés dans la distribution des tâches entre les grandes familles des disciplines scientifiques. Dans un deuxième temps, il s'agira de parcourir deux écrits importants de J. v. Uexküll pour en résumer les principaux enseignements et pointer les proximités importantes avec les théoriciens du pragmatisme. En conclusion, nous verrons comment cet auteur peut nous aider à affiner la définition d'animaux réellement parties prenantes des collectifs d'humains et de non-humains qui prennent forme autour et avec eux.

\section{Le grand partage des animaux}

Un certain nombre d'auteurs l'ont déjà signalé, les animaux tiennent une place particulièrement importante dans les sciences et les philosophies contemporaines

2. J. v. Uexküll, Mondes animaux et monde humain suivi de La théorie de la signification, Paris, Denoël, 90 p. 1965 (c'est nous qui soulignons).

3. On pense par exemple à la manière dont Philippe Descola définit le projet de l'anthropologie : "La mission de celle-ci, telle que je l'entends, est de contribuer avec d'autres sciences, et selon ses méthodes propres, à rendre intelligible la façon dont des organismes d'un genre particulier s'insèrent dans le monde, en acquièrent une représentation stable et contribuent à le modifier en tissant, avec lui et entre eux, des liens constants ou occasionnels d'une diversité remarquable mais non infinie. »P. Descola, Par-delà nature et culture, Paris, Gallimard, 623 p. (2005, p. 12). 
et passées 4 . Pourtant, si à peu près toutes les disciplines se sont saisies des animaux comme objet d'étude, elles l'ont fait chacune de manière très différente en définissant la part du biologiste, la part de l'historien, la part du sociologue, la part du géographe... et c'est ainsi qu'ils se sont retrouvés écartelés sur le chevalet de l'épistémologie moderne. Dans cette partie, nous examinerons, de manière volontairement contrastée (et donc grossière), la manière dont les questions portant sur les animaux sont encore largement distribuées de part et d'autre d'une ligne de partage relativement bien instituée entre les sciences de la nature et les sciences sociales. Nous verrons ensuite en quoi cela nous amène à réinterroger la place des animaux dans les recherches à leur sujet.

\section{Les sciences de la nature : témoins fiables des animaux?}

Nombreuses sont les disciplines ou sous-disciplines pouvant être regroupées dans la grande famille des sciences de la nature. Celles-ci ont beaucoup évolué au cours des derniers siècles, passant par exemple de l'histoire et des sciences naturelles aux sciences de la vie et de l'univers ${ }^{5}$. Cette évolution n'est pas anodine pour aborder la question qui nous occupe mais elle nous informe plus sur la transformation des enjeux sociaux de ces sciences que sur des changements radicaux dans la manière dont elles traitent les animaux. En effet, au cours de l'histoire contemporaine des sciences, le "référentiel animal » n'a que peu évolué. De la génétique à l'éthologie en passant par la physiologie, l'animal-machine, ou du moins l'animal réifié, reste une figure dominante 6 : les animaux restent des entités taxonomiques dont les comportements, les mœurs et l'évolution sont prévisibles dans l'absolu. Le déterminisme régit les sciences de la vie jusque dans l'étude de leurs comportements et la figure de l'animal-machine est poussée à son paroxysme dans certaines manières d'instrumentaliser (au sens propre du terme) certains animaux d'élevage hors-sol ou de laboratoire. Mais les « animaux industriels " ne sont pas les seuls concernés. Brian Wynne montre, par exemple, comment des moutons pâturant à proximité d'une centrale nucléaire se retrouvent transformés par des scientifiques spécialistes de l'énergie nucléaire en de véritables instruments de réponse aux radiations ${ }^{7}$. Le phénomène s'observe également dans nombre de recherches où les animaux ne sont pourtant pas considérés comme de simples moyens servant des finalités productivistes ou scientifiques qui ne les concernent pas directement. J. v. Uexküll

4. B. Cyrulnik, Si les lions pouvaient parler. Essais sur la condition animale, Paris, Gallimard, 1998 ; E. De Fontenay, Le silence des bêtes. La philosophie à l'épreuve de l'animalité, Paris, Fayard, 1998.

5. S. Lambert, «Quand l'écologie et la biologie s'appelaient histoire ou sciences naturelles. Application aux animaux utiles ou nuisible », Le Courrier de l'environnement, vol. 38, 1999.

6. C. Larrère \& R. Larrère, "Actualité de l'animal-machine? ", Sens[public], http:// www.sens-public.org/article.php3?id_article =77, 2004 .

7. B. Wynne, «May the sheep safely graze? A reflexive view of the expert-lay knowledge divide ", in S. Lash, B. Szerszynski \& B. Wynne, eds., Risk, environment and modernity, 1996, pp. 44-83. 
mettait déjà en garde ses lecteurs sur la manière dont ses collègues biologistes essayaient " infatigablement, dans des milliers d'expériences et avec les animaux les plus variés, en commençant par le rat blanc, d'étudier les relations de ces animaux avec un labyrinthe ». Il estimait que "les résultats peu concluants de ces travaux [...], n'importe qui aurait pu les prévoir, à condition de savoir que l'hypothèse implicite sur laquelle ils reposent est erronée : un animal ne peut entrer en relation avec un objet comme tel $\gg$. Cet exemple illustre assez bien la manière dont les sciences de la vie, jusque chez les behaviouristes, ont longtemps négligé le point de vue des animaux sur le monde en réduisant toutes explications possibles du vivant à des mécanismes physico-chimiques.

\section{Les sciences sociales : un rapport abstrait aux animaux}

L'analyse ethnographique des relations entre les hommes et les animaux trouve ses origines chez les folkloristes de la fin du XIXe et le début du XXe siècle, avec notamment les œuvres de Paul Sébillot ${ }^{9}$ et Eugène Rolland ${ }^{10}$. Après Marcel Mauss - qui rappelle que la compréhension d'une société doit comprendre l'étude des animaux et des plantes de celle-ci 11 - et Claude Lévi-Strauss - qui initie un travail de description des modes de catégorisation du vivant 12 -, les relations anthropozoologiques deviennent l'objet d'un grand nombre de recherches dans les années 1970 et 1980, notamment avec l'essor de l'ethnozoologie. En parallèle, la situation dans les pays occidentaux est marquée par un contexte particulier où l'homme parvient plus que jamais à dominer globalement la plupart de ses ennemis naturels et à posséder les technologies permettant une production agricole contrôlée et intensive. Comme pour la sociologie rurale, dont l'émergence en France est marquée par la publication d'un ouvrage 13 qui annonce en même temps la disparition de son objet d'étude 14 , l'engouement suscité par l'analyse des relations hommeanimal au sein des sciences sociales semble contemporain de l'apogée du mythe moderne qui croyait en avoir terminé une bonne fois pour toutes avec la nature et en particulier avec les animaux dont les hommes pensaient s'être émancipés. Les sciences sociales s'intéressant aux relations anthropozoologiques portent ainsi les stigmates de leurs conditions d'émergence en laissant une "nature naturelle » aux sciences de la nature et en traitant avec « sociocentrisme » 15 une nature résorbée

8. J. v. Uexküll (1965), ibid., p. 94.

9. P. Sébillot, Le folklore de France, Paris, Maisonneuve, t. III, 1905.

10. E. Rolland, Faune populaire de la France, Maisonneuve, 13 vol., (1877-1911), Paris, Maisonneuve et Larose, 1967.

11. M. Mauss, Manuel d'ethnographie, Paris, Payot, 1967, p. 62.

12. C. Lévi-Strauss, La pensée sauvage, Paris, Plon, 1962.

13. H. Mendras, La fin des paysans, Paris, Armand Colin, 1964.

14. C. Deverre, "Une rétrospective de la sociologie rurale », Séminaire "Anthropologie, Sociologie et Environnement ", Arles, 2000.

15. C. Larrère \& R. Larrère, Du bon usage de la nature. Pour une philosophie de l'environnement, Paris, Aubier, 1997. 
dans la société : une "nature culturelle ». Si les exemples pouvant illustrer cela sont nombreux, l'analyse des enjeux de "vivre ensemble » autour et avec les grands carnivores sont remarquables à cet égard; comme l'illustre bien cet article traitant de l'alliance entre les sciences sociales et les sciences de la conservation dans une revue naturaliste où nous pouvons lire en exergue, sur fond d'une photographie d'un loup montrant les crocs : "Derrière les mots... des schémas mentaux ».

\section{Brouiller les frontières disciplinaires plutôt que les clarifier}

Les naturalistes peuvent être rassurés car s'ils revendiquent être les spécialistes incontournables des animaux, les sociologues sont pour leur part les spécialistes incontestables des « animots» 16. Le biologiste décrit ce qu'il en est de la nature 17. Le sociologue s'intéresse, quant à lui, à ce que nous pourrions appeler l'" artefact culturel »18. Les comportements humains peuvent alors être expliqués par toute une série de bonnes raisons sociales et culturelles d' " avoir tort » sur la nature et, dans cette histoire, ni les hommes ni les animaux n'y sont vraiment pas pour grandchose. Du point de vue de cette présentation (trop) succincte et (trop) contrastée, le partage entre les sciences de la nature et les sciences sociales apparait comme bien institué et cette distribution toute faite des tâches ne rend pleinement justice ni aux animaux des biologistes, ni aux humains des socio-anthropologues. L'enjeu n'est donc pas tant de consolider un mode d'articulation des positionnements scientifiques basé sur un partage profondément établi au détriment des objets analysés. Il s'agit plutôt - dans la lignée du projet de l'anthropologie symétrique 19 et de l'anthropologie de la nature 20 - de rompre avec un certain confort épistémologique en brouillant les lignes de positionnement actuelles afin de voir dans quelle mesure il est possible de mieux traiter les hommes et les animaux dans l'analyse socio-anthropologique des relations anthropozoologiques. Nous allons voir dans la suite de cet article comment $\mathrm{J}$. v. Uexküll peut nous aider dans la poursuite d'un tel exercice.

\section{Recomposer (avec) les animaux}

Les questions du langage, des techniques et de la conscience animale ont longtemps occupé une place considérable dans les recherches sur les animaux, avec en

16. Selon de mot de Derrida, dans J. Derrida, L'animal que donc je suis, Paris, Galilée, 2006.

17. Pour reprendre l'exemple des loups, il explique la biologie du loup, l'état favorable de conservation de ses populations, etc.

18. Dans le cadre de notre exemple, la peur des loups, les problèmes d'acceptation sociale dont ils sont victimes, etc.

19. Notamment B. Latour, Nous n'avons jamais été modernes. Essai d'anthropologie symétrique, Paris, La Découverte, 1991.

20. Notamment P. Descola (2005). 
arrière-fond une même finalité : tenter de distinguer le propre de l'homme du propre de l'animal. Ces recherches se sont développées aux dépens de la question des compétences sémiotiques des animaux et J. v. Uexküll est sans doute l'un des premiers scientifiques à s'être intéressé aux mondes vécus de ces derniers. Souvent désigné comme l'un des grands précurseurs de l'écologie et plus spécifiquement de l'éthologie contemporaine, cet auteur a également été l'objet de lectures attentives et d'inspirations de la part d'un certain nombre de philosophes ${ }^{21}$. Nous proposons pour notre part de voir comment il peut nous aider à nous ressaisir de la question des relations anthropozoologiques dans le cadre d'une sociologie d'inspiration pragmatiste. Jacob von Uexküll (1864-1944) est un naturaliste et biologiste allemand. Étudiant plus particulièrement la zoologie, ses premiers travaux portent sur la physiologie des invertébrés. Il mène alors des recherches pouvant être qualifiées de mécanistes et se fait "l'avocat d'une psychologie animale désinfectée de tout anthropomorphisme » 22 . Il va ensuite s'intéresser à la physiologie musculaire et alors que ses recherches le conduisent au plus près des explications physico-chimiques du vivant - il est amené à repenser l'organisme dans sa totalité et dans l'ensemble de ses relations aux milieux concrets. J. v. Uexküll est l'auteur d'un très grand nombre de publications scientifiques et de vulgarisation, peu traduites et rééditées. Nous nous concentrerons ici sur ce qui restera sans doute ses deux principaux écrits dans lesquels il développe sa théorie de l'Umwelt 23 : Mondes animaux et monde humain et Théorie de la signification. J. v. Uexküll y souligne l'importance des mondes vécus, qu'il décompose en un double système - celui du Merkwelt (le monde perceptif) et celui du Wirkwelt (le monde actif) - conçu moins comme un support explicatif que comme un outil descriptif permettant de structurer et d'organiser l'observation rigoureuse des mondes concrets. Si nous avions à résumer le projet de J. v. Uexküll, nous pourrions dire qu'il cherche à nous donner une chance de porter le regard dans le monde vécu singulier des multiples êtres vivants. Car, nous dit le biologiste, chaque organisme vit dans un monde propre auquel il est possible d'accéder par l'intermédiaire de ses sens. Il ne s'agit pas bien sûr de se mettre à la place d'un bourdon, d'une chauve-souris ou même d'un chimpanzé, mais d'améliorer la compréhension des règles de perception et d'action qui président à la constitution des liens qu'ils tissent avec ce qui les entoure 24 . L'exemple qu'il prend de la tique illustre cette proposition :

21. Citons notamment M. Heidegger, Les concepts fondamentaux de la métaphysique, Paris, Gallimard, 1992 ; M. Merleau-Ponty, La Nature. Notes du Cours du Collège de France, Paris, Seuil, 1956-1957 ; P. Sloterdijk, Écumes. Sphères III, Paris, Maren Sell, 2005.

22. P. Muller (1965). Présentation de «Mondes animaux et monde humain suivi de La théorie de la signification ", in J. v. Uexküll (1965), op. cit., p. 7.

23. Ce terme allemand est traduit de différentes manières selon les auteurs : environnement, milieu, entourage. Il semble que la traduction la mieux adaptée pour traduire la pensée de J. v. Uexküll serait celle d' " entour».

24. L'exemple que J. v. Uexküll nous donne du gourmet illustre bien cela : «Il ne nous intéresse pas de savoir quelles sensations gustatives raisins et fruits confits réservent au gourmet; 
Cet animal, privé d'yeux, trouve le chemin de son poste de garde à l'aide d'une sensibilité générale de la peau à la lumière. Ce brigand de grand chemin, aveugle et sourd, perçoit l'approche de ses proies par son odorat. L'odeur de l'acide butyrique, que dégagent les follicules sébacés de tous les mammifères, agit sur lui comme un signal qui le fait quitter son poste de garde et se lâcher en direction de sa proie. S'il tombe sur quelque chose de chaud (ce que décèle pour lui un sens affiné de la température), il a atteint sa proie, l'animal à sang chaud, et n'a plus besoin que de son sens tactile pour trouver une place aussi dépourvue de poils que possible, et s'enfoncer jusqu'à la tête dans le tissu cutané de celle-ci. Il aspire alors lentement à lui un flot de sang chaud 25.

On découvre ici les aptitudes sémiotiques de cet acarien et la manière dont celuici réduit la complexité du réel dans laquelle il baigne pour y ajuster son existence sur la simple base de trois signaux (température, acide butyrique et densité de poils). Comme tout organisme, la tique " enserre son objet dans les deux branches d'une pince - une branche perceptive et une branche active » 26 . Cet ajustement combinatoire entre un porteur de caractères actifs et perceptifs (le mammifèreobjet) et un récepteur de significations (la tique-sujet) peut alors être représenté comme la succession de trois cercles fonctionnels qui supposent à chaque fois, pour la tique, l'utilisation de moyens spécifiques pour agir et pour percevoir. L'un des enseignements importants de la théorie de l'Umwelt est de pointer le rôle prépondérant de l'action comme moyen, pour le sujet, de doter ce qui l'entoure de formes significatives. L'univers n'a donc pas de sens « en soi ». Les significations doivent être instaurées par l'articulation d'objets porteurs de significations et de sujets dotés de moyens d'action et de perception. Pour prendre un autre exemple simple de J. v. Uexküll, la construction de la toile est pour l'araignée la mise en ceuvre de la signification "proie» pour la mouche. J. v. Uexküll propose alors d'élaborer une théorie de la composition naturelle en analogie au modèle de la théorie de la composition musicale. Comme dans un duo où les deux voix doivent se correspondre note pour note, point pour point, pour être en harmonie ${ }^{27}$, deux êtres vivants s'ajustent l'un à l'autre en entretenant des rapports contrapuntiques. L'exemple de la tique permet à nouveau d'illustrer cette règle de signification contrapuntique (tableau 1).

nous constatons seulement qu'ils deviennent des caractères perspectifs de son milieu, parce qu'ils ont pour lui une signification biologique particulière » (J. v. Uexküll, 1965, op. cit., p. 28) 25. Ibid., p. 18.

26. Ibid., p. 23.

27. Ibid., p. 131. 
Tableau 1 : Rapport contrapuntique entre une tique et un mammifère 28

\begin{tabular}{|l|l|}
\hline \multicolumn{1}{|c|}{$\begin{array}{c}\text { Point : tique récepteur } \\
\text { de signification }\end{array}$} & $\begin{array}{l}\text { Contrepoint : mammifère quelconque } \\
\text { porteur de signification }\end{array}$ \\
\hline $\begin{array}{l}\text { 1. Lodorat est réglé sur une seule odeur, } \\
\text { celle de l'acide butyrique. }\end{array}$ & $\begin{array}{l}\text { 1. La seule odeur que les mammifères ont } \\
\text { en commun est l'acide butyrique de leur } \\
\text { transpiration. }\end{array}$ \\
\hline $\begin{array}{l}\text { 2. Présence d'un organe tactile qui permet } \\
\text { à la tique de s'orienter parmi les poils de la } \\
\text { proie. }\end{array}$ & 2. Les mammifères ont des poils. \\
\hline $\begin{array}{l}\text { 3. Organe thermomètre signalant le } \\
\text { chaud. }\end{array}$ & 3. Les mammifères ont une peau chaude. \\
\hline $\begin{array}{l}\text { 4. Rostre permettant de perforer la peau } \\
\text { des mammifères et servant en même } \\
\text { temps à pomper un liquide. }\end{array}$ & $\begin{array}{l}\text { 4. Les mammifères ont une peau molle } \\
\text { parcourue de nombreux canaux sanguins. }\end{array}$ \\
\hline
\end{tabular}

Les organismes sont donc accordés les uns avec les autres dans un "orchestre naturel » 29. C'est là, nous semble-t-il, toute la radicalité des théories uexkülliennes qui tentent de libérer la biologie de la recherche des déterminations causales pour mettre au premier plan la compréhension des relations de signification. Cette manière d'observer et de décrire les mondes animaux amène le biologiste à livrer une lecture pluraliste du réel. Il nous montre de manière éloquente comment chaque entité est prise comme objet dans une multitude de mondes vécus. La citation qu'il propose d'un livre de Werner Sombart, De l'homme, illustre bien cela : «Il n'existe pas de forêt en tant que milieu objectivement déterminé il y a une forêtpour-le-forestier, une forêt-pour-le-chasseur, une forêt-pour-le-botaniste, une forêt-pour-le-promeneur, une forêt-pour-l'ami-de-la-nature, une forêt-pour-celuiqui-ramasse-du-bois ou celui-qui-cueille-des-baies, une forêt de légende où se perd le petit poucet » 30 . J. v. Uexküll décrit donc les choses dans leur pluralité et met en garde contre les attitudes qui consistent à faire la présentation d'un monde univoque en adoptant un point de vue surplombant sur les mondes à partir de catégories conceptuelles abstraites. Mais à ce stade, un problème délicat subsiste : comment la mélodie d'un univers aussi fourmillant peut-elle être harmonieuse ? Tel est le dilemme pluraliste auquel J. v. Uexküll tente d'échapper en passant brutalement de la description méticuleuse du concret à l'explication rapide et abstraite du tout. En conclusion de son ouvrage, Mondes animaux et monde humain, il invoque en effet «la nature » comme sujet absolu qui lui permet de faire tenir le foisonnement des mondes vécus dans un tout harmonieux : « [...] tous ces milieux sont portés et conservés par la totalité qui transcende chaque milieu particulier. Derrière tous les mondes auxquels il donne naissance, se cache, éternellement

28. D'après Uexküll, ibid., p. 138.

29. Ibid., p. 151.

30. Sombart, cité dans J. v. Uexküll, ibid., p. 97. 
présent, le sujet : la nature » 31 . En faisant de James un « lecteur anachronique » de J. v. Uexküll, Vinciane Despret et Stéphan Galetic 32 montrent comment le pragmatisme aurait pu permettre au biologiste de ne pas bafouer son amour du concret en résolvant trop vite un tel dilemme pluraliste. Pour les pragmatistes, en effet, si le vrai n'existe qu'à travers l'intérêt d'un sujet sur le monde, une théorie de la vérité ne peut se constituer qu'à travers l'idée d'une communauté de vies singulières assemblées, d'une harmonie des personnes ${ }^{33}$. La proximité d'une telle conception avec la théorie de la composition naturelle de J. v. Uexküll est frappante. Pourtant, un point important les différencie : la tenacité à tenir l'expérience empirique jusque dans la tentative d'une description de l'unification de la pluralité. V. Despret et $\mathrm{S}$. Galetic parviennent à prolonger l'exercice de description de J. v. Uexküll en maintenant obstinément leur attention sur les expériences concrètes qui participent de la composition d'un monde commun. Avec l'aide de James, ils nous invitent à éviter tout recours à l'abstraction en refusant de lâcher prise trop vite sur les faits. Car pour l'auteur des Essais d'empirisme radical 34 , le fait c'est ce qui donne prise à l'analyse, ce à quoi le philosophe doit savoir s'accrocher sans craindre que celui-ci ne résiste pas à sa propre expérience. Les faits constituent des prises fiables sur lesquelles les observateurs doivent savoir compter pour « dépouiller le monde objectif de son étrangeté, et [...] faire que nous nous y sentions plus chez nous » 35 . Comme le soulignent V. Despret et S. Galetic, cette obstination pour les faits suppose de redonner à notre corps la confiance qu'il mérite en "abandonnant les concepts pour le chaos de la vie réelle » 36 : «le monde dont on fait l'expérience [...] se présente avec notre corps pour centre, centre de vision, centre d'action, centre d'intérêt [...] tout tourne autour de lui et est ressenti de ce point de vue »37. Par ce qu'ils appellent un perspectivisme radical, ils nous exhortent alors à "résister au choix combinatoire entre un monde qui existe "par nous" [auquel la perspective sociocentrique que nous avons décrite plus haut contribue parfaitement] et un monde qui existe "malgré nous" [auquel la perspective biocentrique que nous avons décrite plus haut contribue parfaitement]. Le perspectivisme radical nous propose un monde "avec nous" » 38 .

31. Ibid., p. 90.

32. V. Despret \& S. Galetic, «Faire de James un lecteur anachronique de von Uexküll : esquisse d'un perspectivisme radical ", in D. Debaise, éd., Vie et expérimentation. Peirce, James, Dewey, Bruxelles, Vrin, 2007, pp. 45-75.

33. J. Wahl, Les philosophies pluralistes d'Angleterre et d'Amérique, Paris, Les Empêcheurs de penser en rond, 2005, p 132.

34. W. James, Essais d'empirisme radical, Paris, Agone, 2005.

35. James (se référent à Hegel) in W. James, A pluralistic universe. Hibbert lectures at Manchester College on present situation in philosophy, Londres, Bombay et Calcutta, Longmans, Green and Co, 1909, p. 11 (cité par V. Despret \& S. Galletic (2007), ibid., p. 45.

36. W. James (1909), ibid., p. 256 (cité par V. Despret \& S. Galletic (2007), ibid., p. 68). 37. W. James, Essays in Radical Empiricism, Longmans, Green and Co., New York, Bombay et Calcutta, 1912, p. 168-171 (cité par V. Despret \& S. Galletic (2007), ibid., p. 58).

38. V. Despret \& S. Galetic (2007), ibid., p. 70. 


\section{Quelle place pour des animaux qui compteraient?}

Si nous acceptons la proposition de V. Despret et de S. Galetic, alors se pose la question de la composition de ce "nous", cette question sur laquelle Isabelle Stengers 39 ou Donna Haraway 40 nous interpellent si efficacement. Dans la lignée des études relativement récentes reconnaissant aux animaux un statut d'êtres relationnels 41 , l'enjeu est de poursuivre l'ouverture du « nous » à ces non-humains en leur reconnaissant la place entière d'actant et d'interagissant qu'ils méritent.

Nous avons vu dans le début de ce texte que les animaux étaient encore essentiellement considérés soit dans une perspective biocentrique - où les déterminants biologiques et physico-chimiques constituent la base de compréhension de l'animal -, soit dans une perspective sociocentrique - où la société humaine tend à constituer l'unique référentiel de problématisation. Comment s'y prendre alors pour dépasser cela et conduire concrètement notre enquête sur les relations anthropozoologiques dans la lignée du perspectivisme radical? Ces dernières années quelques sociologues et anthropologues ont tenté de répondre à cette question en mettant en place des dispositifs d'observation adaptés aux mondes humains et animaux qu'elles 42 étudiaient. Florence Brunois explique comment elle mène son étude auprès des Kasuas de Papouasie Nouvelle-Guinée en présence de la plante ou de l'animal pour garantir «la prise en considération du/des comportement(s) agissant(s) de l'être vivant et de l'observateur humain et d'isoler ainsi les divers processus gouvernant la reconnaissance, la nomination comme la catégorisation de l'être rencontré, et au-delà, les discontinuités qu'établissent éventuellement les populations entre ces êtres » 43 . Joceline Porcher ou Marion Vicart vont encore plus loin. La première analyse par exemple les relations entre des éleveurs et des animaux d'élevage en " constituant un "questionnaire animal" destiné à appréhender le "point de vue" de l'animal sur son monde et sa relation à

39. I. Stengers, La vierge et le neutrino, Paris, Les Empêcheurs de penser en rond, 2006. 40. D. Haraway, Simians, Cyborgs and Women: The Reinvention of Nature, New York, 1991.

41. F. De Waal, De la réconciliation chez les primates, Paris, Flammarion, 1992 ; V. Despret, Naissance d'une théorie éthologique. La danse du cratérope écaillé, Paris, Les Empêcheurs de penser en rond, 1996 ; V. Despret, "Sheep do have opinions ", in B. Latour \& P. Weibel, eds., Making things public. Atmospheres of democracy, Cambridge, Massachusetts, The MIT Press, 2005, pp. 360-368 ; J. Goodall, «Intercommunity interactions in the chimpanzee population of the Gombe National Park », in W.C. McGrew, L.F. Marchant \& T. Nishida, eds., Great ape societies, Cambridge, Cambridge University Press, 1979; D. Lestel, Les origines animales de la culture, Paris, Flammarion, 2003 ; S.C. Strum \& B. Latour, "Redifining the social link: from baboons to humans", Social Sciences Informations, vol. 26(4), 1987. pp. 783-802.

42. Il est flagrant de constater à quel point les femmes constituent la très large majorité des " chercheurs de l'animal relationnel ». Cela est vrai aussi bien pour les éthologues, les philosophes, les sociologues, les anthropologues ou les géographes.

43. F. Brunois, "Pour une approche interactive des savoirs locaux : l'ethno-éthologie ", Journal de la Société des Océanistes, vol. 120, 2005, pp. 31-40. 
l'éleveur »44. La seconde développe une phénoménographie équitable qui ambitionne de suivre les animaux dans leur « dynamique ontologique » en décrivant les êtres humains et animaux "dans la pluralité de leur mode d'existence "sans "s'arrêter sur les moments (clefs) où les seconds sont utiles à la mobilisation des compétences des premiers » 45 . Mais comment faire pour prendre au sérieux la part des animaux rétifs, quasiment invisibles, qui ne laissent que peu de prises aux entités qu'ils affectent? Telle est certainement une difficulté face à laquelle les innovations méthodologiques citées précédemment ne sont que d'une utilité limitée. Impossible en effet de mener, par exemple, des entretiens en présence d'animaux aussi discrets que les loups qui peuplent l'Arc Alpin. Néanmoins, beaucoup d'objets du monde vécu de ces prédateurs sont directement accessibles pour l'enquêteur et peuvent porter les traces d'une expérience avec un loup ${ }^{46}$. La question se pose alors de savoir dans quelle mesure il est possible de compter sur ces objets du monde vécu des loups comme témoins fiables de ces animaux. Pour revenir à un exemple cher à J. v. Uexküll, il s'agit de se demander si l'on peut compter sur le mammifère pour comprendre la tique comme sujet? Même si les expériences directes de loups sont relativement rares comparées à l'étendue des collectifs qu'ils affectent, leur récalcitrance est mise à l'épreuve d'un certain nombre d'expériences concrètes et de traces d'expériences directes qui fournissent malgré tout à l'enquêteur des prises concrètes sur ces animaux. Pister leurs traces dans la neige, poser des filets de protection contre les attaques des moutons, faire l'analyse génétique de leurs fèces, accompagner un groupe de touristes sur le territoire d'une meute, instruire les dossiers d'indemnisation des brebis tuées par les loups, assister à une soirée débat sur la protection des grands carnivores, être poursuivi en justice pour destruction d'une espèce protégée..., autant d'expériences concrètes d'interenrôlement entre des loups et des humains à travers une série de médiateurs qui nous informent sur ces loups aux prises avec des humains et sur ces humains aux prises avec des loups. Il s'agit donc de focaliser notre attention sur les expériences rarement directes mais toujours concrètes de ces animaux qui permettent d'accéder aux loups comme objets des mondes humains et non humains et, dans une moindre mesure, aux loups comme sujets par l'analyse des stigmates qu'ils laissent sur les objets qui composent leurs mondes vécus.

44. J. Porcher, L'élevage, un partage de sens entre hommes et animaux : intersubjectivité des relations entre éleveurs et animaux dans le travail, Paris, Institut National Agronomique Paris-Grignon, 2001, p. 160. Jocelyne Porcher propose une observation vidéo standardisée pour compléter ses questionnaires auprès des éleveurs. Elle filme successivement un plan d'ensemble des lieux où sont les animaux, trois plans successifs de trois animaux différents spatialement situés à des endroits différents, et enfin l'éleveur avec ces animaux.

45. M. Vicart, «Regards croisés entre l'animal et l'homme : petit exercice de phénoménographie équitable », Ethnographiques.org, vol. 17, 2008. http://www.ethnographiques.org/ 2008/Vicart.

46. Pour une étude de cas, voir notamment A. Doré, "Le devenir politique des corps recomposés. La circulation des animaux dans l'espace public ", Sociologie et Sociétés (2010, à paraître). 
Ainsi le socio-anthropologue n'est pas contraint de rechercher dans l'homme toutes les explications des relations anthropozoologiques. Les animaux concrets ne sont plus l'exclusivité du biologiste et ils deviennent un facteur important de compréhension de la forme des collectifs et des liens qui les font tenir. J. v. Uexküll nous rappelle que les animaux sont dotés de qualités singulières, d'expériences, de compétences sémiotiques et qu'il existe pour eux aussi des choses " qui comptent » et qui « ne se valent pas » 47 . Ils sont à la fois porteurs et récepteurs de caractères perceptifs et actifs, et leur existence dans les collectifs est donc portée à la fois par chacun des membres du collectif (les animaux comme objets dans le monde des autres-sujets) et par les animaux eux-mêmes (les autres comme objets dans le monde des animaux-sujets).

\section{Références bibliographiques}

F. Brunois, "Pour une approche interactive des savoirs locaux : l'ethno-éthologie ", Journal de la Société des Océanistes, vol. 120, 2005. pp. 31-40.

B. Cyrulnik, Si les lions pouvaient parler. Essais sur la condition animale, Paris, Gallimard, 1998.

J. Derrida, L'animal que donc je suis, Paris, Galilée, 2006.

P. Descola, Par-delà nature et culture, Paris, Gallimard, 2005.

V. Despret, Naissance d'une théorie éthologique. La danse du cratérope écaillé, Paris, Les Empêcheurs de penser en rond, 1996.

V. Despret, Quand le loup habitera avec l'agneau, Paris, Les Empêcheurs de penser en rond, 2002.

V. Despret, "Sheep do have opinions ", in B. Latour \& P. Weibel, éds, Making things public. Atmospheres of democracy, Cambridge, Massachusetts, The MIT Press, 2005. pp. 360-368.

V. Despret, et S. Galetic, "Faire de James un lecteur anachronique de von Uexküll : esquisse d'un perspectivisme radical ", in D. Debaise, éd., Vie et expérimentation. Peirce, James, Dewey, Bruxelles, Vrin, 2007. pp. 45-75.

C. Deverre, "Une rétrospective de la sociologie rurale", Séminaire "Anthropologie, Sociologie et Environnement »: Arles, 2000.

A. Doré, «Le devenir politique des corps recomposés. La circulation des animaux dans l'espace public », Sociologie et Sociétés (2010, à paraître).

E. De Fontenay, Le silence des bêtes. La philosophie à l'épreuve de l'animalité, Paris, Fayard, 1998.

J. Goodall, "Intercommunity interactions in the chimpanzee population of the Gombe National Park », in W.C. McGrew, L.F. Marchant \& T. Nishida, eds., Great Ape Societies, Cambridge, Cambridge University Press, 1979.

D. Haraway, Simians, Cyborgs and Women : The Reinvention of Nature, New York, 1991.

M. Heidegger, Les concepts fondamentaux de la métaphysique, Paris, Gallimard, 1992.

W. James, A pluralistic universe. Hibbert lectures at Manchester College on present situation in philosophy, Londres, Bombay et Calcutta, Longmans, Green and Co, 1909.

47. Sur les manières d'explorer en éthologie ce qui importe aux animaux, voir notamment V. Despret, Quand le loup habitera avec l'agneau, Paris, Les Empêcheurs de penser en rond, 2002. Pour une présentation plus générale de cette philosophie de l'attention à «ce qui importe » aux autres, voir I. Stengers (2006). 
W. James, Essays in Radical Empiricism, Longmans, Green and Co., New York, Bombay \& Calcutta, 1912.

W. James, Essais d'empirisme radical, Paris, Agone, 2005.

S. Lambert, "Quand l'écologie et la biologie s'appelaient histoire ou sciences naturelles. Application aux animaux utiles ou nuisible ", Le Courrier de l'environnement, vol. 38, 1999.

C. Larrère \& R. Larrère, Du bon usage de la nature. Pour une philosophie de l'environnement, Paris, Aubier, 1997.

C. Larrère \& R. Larrère, «Actualité de l'animal-machine ? », Sens[public], http://www.senspublic.org/article.php3?id_article=77, 2004.

B. Latour, Nous n'avons jamais été modernes. Essai d'anthropologie symétrique, Paris, La Découverte, 1991.

D. Lestel, Les origines animales de la culture, Paris, Flammarion, 2003.

C. Lévi-Strauss, La pensée sauvage, Paris, Plon, 1962.

M. Mauss, Manuel d'ethnographie, Paris, Payot, 1967.

H. Mendras, La fin des paysans, Paris, Armand Colin, 1964.

M. Merleau-Ponty, La Nature. Notes du Cours du Collège de France, Paris, Seuil, 1956-1957.

J. Porcher, L'élevage, un partage de sens entre hommes et animaux : intersubjectivité des relations entre éleveurs et animaux dans le travail, Paris, Institut National Agronomique Paris-Grignon, 2001.

E. Rolland, Faune populaire de la France, Maisonneuve, 13 vol., (1877-1911). Paris, Maisonneuve et Larose, 1967.

P. Sébillot, Le folklore de France, Paris, Maisonneuve, t. III. 1905.

P. Sloterdijk, Écumes. Sphères III, Paris, Maren Sell, 2005.

I. Stengers, La vierge et le neutrino, Paris, Les Empêcheurs de penser en rond, 2006.

S.C. Strum \& B. Latour, "Redifining the social link: from baboons to humans ", Social Sciences Informations, vol. 26(4), 1987. pp. 783-802.

J. v. Uexküll, Mondes animaux et monde humain suivi de La théorie de la signification, Paris, Denoël, 1965.

M. Vicart, «Regards croisés entre l'animal et l'homme : petit exercice de phénoménographie équitable », Ethnographiques.org, vol. 17, 2008. http://www.ethnographiques.org/ 2008/Vicart

F. De Waal, De la réconciliation chez les primates, Paris, Flamarion, 1992.

J. Wahl, Les philosophies pluralistes d'Angleterre et d'Amérique, Paris, Les Empêcheurs de penser en rond, 2005.

B. Wynne, "May the sheep safely graze? A reflexive view of the expert-lay knowledge divide ", in S. Lash, B. Szerszynski \& B. Wynne, eds., Risk, environment and modernity, 1996, pp. 44-83. 